\title{
COVID-19 and primary care physicians: adapting to rapid change in clinical roles and settings
}

\author{
Alden Yuanhong Lai ${ }^{1 \wedge}$, Samuel C. Thomas ${ }^{2,3}$, Erin E. Sullivan ${ }^{4,5}$, Bram P. I. Fleuren $^{6}$, Minakshi Raj $^{7}$, \\ Matthew J. DePuccio ${ }^{8}$, Amber L. Stephenson ${ }^{9}$, Ann Scheck McAlearney ${ }^{8,10}$ \\ ${ }^{1}$ Department of Public Health Policy and Management, School of Global Public Health, New York University, New York, NY, USA; ${ }^{2}$ Department \\ of Primary Care and Population Health, Stanford University School of Medicine, Stanford, CA, USA; ${ }^{3}$ Intermountain Healthcare Delivery Institute, \\ Intermountain Healthcare, Salt Lake City, UT, USA; ${ }^{4}$ Center for Primary Care, Harvard Medical School, Boston, MA, USA; ${ }^{5}$ Sawyer Business \\ School, Suffolk University, Boston, MA, USA; ${ }^{6}$ Department of Work and Social Psychology, Faculty of Psychology and Neuroscience, Maastricht \\ University, Maastricht, The Netherlands; ${ }^{7}$ Department of Kinesiology and Community Health, University of Illinois at Urbana Champaign, \\ Champaign, IL, USA; ${ }^{8}$ CATALYST, Center for the Advancement of Team Science, Analytics, and Systems Thinking, College of Medicine, The Ohio \\ State University, Columbus, OH, USA; ${ }^{9}$ David D. Reh School of Business, Clarkson University, Schenectady, NY, USA; ${ }^{10}$ Department of Family and \\ Community Medicine, College of Medicine, The Ohio State University, Columbus, OH, USA \\ Correspondence to: Alden Yuanhong Lai, PhD, MPH. New York University School of Global Public Health, 715 Broadway, $10^{\text {th }}$ Floor, \#1000A, New \\ York, NY 10003, USA. Email: aldenlai@nyu.edu.
}

Received: 10 September 2020. Accepted: 10 October 2020; Published: 25 December 2020.

doi: $10.21037 /$ jhmhp-20-130

View this article at: http://dx.doi.org/10.21037/jhmhp-20-130

With the emergence of COVID-19, drastic measures have been taken to create, deploy, and sustain a pandemic workforce as well as to continuously adapt this workforce in preparation for future needs (1). One major shift in primary care has been to deliver care via telemedicine. Another shift that has been more disruptive to primary care practice patterns, however, is the deployment of primary care physicians (PCPs) to hospitals in order to meet emergent care delivery needs. In many hospital and health systems, PCPs have been expected to join the hospital-based workforce and take on roles in emergency, hospitalist, and respiratory medicine as the pandemic has overwhelmed and depleted many hospitals' existing workforce capacities. This article highlights challenges in such deployment efforts and proposes recommendations to help PCPs and health care organizations adapt so that they are able to effectively respond to potential upcoming pandemic waves.

\section{Challenges with deploying PCPs to inpatient care settings}

First, the speed and magnitude of changes to which PCPs have had to react and respond as they became a part of many hospitals' pandemic workforces had notable impacts on both their professional and personal wellbeing. In many cases, PCPs experienced early anxiety at the very prospect of having to function as inpatient clinicians, as they may have lacked up-to-date training and the skills necessary to deliver comprehensive and competent inpatient care given many PCPs had not provided hospital-based care since they had completed their residency training (2). This issue was particularly salient in health systems that had fully adopted a hospitalist model.

Second, PCPs entering the inpatient workforce have had to navigate different workflows, protocols, equipment, team compositions, and cultures unique to the hospital

$\wedge$ ORCID: 0000-0002-2416-2915. 
environment. For instance, PCPs may have been unfamiliar with dynamic protocols for admitting and discharging patients in hospital settings, and they may have had limited experience using inpatient electronic health record systems. Furthermore, in many hospitals, these PCPs may not have had the appropriate permissions to access the electronic records required to deliver and document inpatient care. Such organizational and operational challenges may have decreased these providers' perceived self-efficacy with respect to their ability to participate in hospital-based care, and may have further contributed to PCPs' levels of distress and potentially impacting work performance (3).

Third, as the work hours and days that are typical of hospital shift work can result in sleep loss and fatigue, this change in work expectations may have also negatively impacted PCPs' well-being (4). Sleep deprivation, keeping excessively long hours, and insufficient recovery has threatened clinician well-being by creating and perpetuating burnout conditions among physicians (5). Given that burnout has recently been found to affect more than $31 \%$ of PCPs $(6,7)$. this is of particular concern in the context of organizations' responses to COVID-19.

\section{Recommendations for health care organizations}

Given the aforementioned challenges associated with transitioning $\mathrm{PCPs}$ into inpatient environments and considering lessons learned from the first few months of the pandemic, we present several recommendations for health care organizations attempting to prepare for future changes in care delivery processes. First, although PCPs responding to the needs of a disease such as COVID-19 may not be immediately asked to run ventilators upon joining the hospital-based workforce, hospitals will benefit by anticipating and eliminating challenges associated with preparing PCPs for a transition to inpatient care. For instance, PCPs introduced to the hospital setting will have to coordinate work with and rely on new team members as they navigate the unfamiliar landscape of inpatient units. This will be particularly important as recent research has shown that perceived teamwork efficiency is significantly associated with reduced odds of burnout and may increase physician well-being (8). Embracing the tenets of team-based care-trust building, task delegation, and open communication-can increase PCPs' comfort with delivering hospital-based care as they respond to rapidly changing circumstances such as those required by COVID-19. Leveraging the skills and competencies of different team members is particularly helpful to PCPs as more collaborative care has been found to facilitate better decision-making and allow teams to better address inpatients' needs (9). Furthermore, ensuring that there are systematic processes by which PCPs can gain access to and learn how to use the tools used to deliver inpatient care will empower and prepare them to be comfortable and proficient team members upon deployment.

In addition, the emergence of COVID-19 has shown that PCPs will need assistance orienting to the different roles and responsibilities that exist on inpatient wards so that they can efficiently assist in this new context. An example from the UK shows how a COVID-19 cross-skilling training program was developed to reacquaint doctors with inpatient practice; this program included content on personal protective equipment (PPE) use, intensive care scenarios, diagnostics, and resuscitation simulations (10). Hospital administrators should consider arranging similar training or orientation sessions, either in person or virtually, to introduce PCPs to their new colleagues and review important policies and procedures relevant to their particular hospital settings. Orientation sessions can also provide opportunities for PCPs to interact with floor or nurse managers to learn about how shifts are managed, and about the roles of different inpatient team members whom they may encounter during their shifts. Prior research has shown that healthcare professionals on the front lines value such acts of solidarity, especially during challenging times (2). Operationalizing this type of orientation activity can increase PCPs' confidence about providing inpatient care and help them to both develop rapport with new team members and learn where to safely seek help as they adjust to the demands of inpatient settings.

A third opportunity is to match inpatient providers such as hospitalists, residents, or advanced practice professionals (APPs) with PCPs who will be joining the hospital-based workforce during the transition period. Research has shown that intentionally linking incoming PCPs to clinicians who have ample experience in the inpatient setting can promote learning and prevent clinician burnout (11). However, implementing such an approach requires several considerations. First, provider characteristics such as experience, competencies, work schedules, and existing social connections should be taken into account when pairing providers as many of these factors are predictive of high-quality relationships (12). Second, hospitals may need to create opportunities for incoming PCPs to shadow their inpatient partner for a specific time frame (e.g., a week); if 
there is sufficient time, roles can then be reversed to foster additional learning and build PCPs' confidence about their ability to contribute in the hospital setting (13). Third, the partnership match should ideally be maintained for the duration of the altered working arrangement, and perhaps even beyond. This longer-term perspective can ensure PCPs retain meaningful access to a trusted colleague as well as social support, and these factors can also reduce the anxiety associated with their being in a new role (11). Throughout this process, adequate organizational support will be necessary, including recognizing that increased workload will be involved for those inpatient partners accommodating new PCP colleagues. Nonetheless, dedicated transition time will prove invaluable to PCPs with respect to their ability to adjust to new roles and meaningfully contribute to hospitalbased care.

Maintaining a sustainable health workforce is crucial in responding to the COVID-19 pandemic. Yet, the initial lessons learned about redeploying clinicians to meet the needs of this crisis highlight the necessity to consider ways to proactively address the challenges associated with such rapid role changes. Hospitals are ideally positioned to help reduce PCPs' anxiety and ensure adequate preparation for their new roles in unfamiliar clinical settings. Considering these recommendations can help to reduce inefficiencies as PCPs are reassigned to work with existing, stressed clinical teams caring for the critically ill. Facilitating PCPs' transition to inpatient care can enhance our collective efforts to manage care needs during the present COVID-19 pandemic, as well as prepare for future public health crises.

\section{Acknowledgments}

Funding: None.

\section{Footnote}

Provenance and Peer Review: This article was commissioned by the editorial office, Fournal of Hospital Management and Health Policy for the series "Innovations and Practices that Influence Patient-Centered Health Care Delivery". The article did not undergo external peer review.

Conflicts of Interest: All authors have completed the ICMJE uniform disclosure form (available at http://dx.doi. org/10.21037/jhmhp-20-130). The series "Innovations and Practices that Influence Patient-Centered Health Care Delivery" was commissioned by the editorial office without any funding or sponsorship. Ann Scheck McAlearney served as the unpaid Guest Editor of the series. The authors have no other conflicts of interest to declare.

Ethical Statement: The authors are accountable for all aspects of the work in ensuring that questions related to the accuracy or integrity of any part of the work are appropriately investigated and resolved.

Open Access Statement: This is an Open Access article distributed in accordance with the Creative Commons Attribution-NonCommercial-NoDerivs 4.0 International License (CC BY-NC-ND 4.0), which permits the noncommercial replication and distribution of the article with the strict proviso that no changes or edits are made and the original work is properly cited (including links to both the formal publication through the relevant DOI and the license). See: https://creativecommons.org/licenses/by-nc-nd/4.0/.

\section{References}

1. Fraher EP, Pittman P, Frogner BK, et al. Ensuring and Sustaining a Pandemic Workforce. N Engl J Med 2020;382:2181-3.

2. Shanafelt T, Ripp J, Trockel M. Understanding and Addressing Sources of Anxiety Among Health Care Professionals During the COVID-19 Pandemic. JAMA 2020;323:2133-4.

3. Shoji K, Cieslak R, Smoktunowicz E, et al. Associations between job burnout and self-efficacy: a meta-analysis. Anxiety Stress Coping 2016;29:367-86.

4. Mallis MM. Fatigue challenges in emergency medical services operations. Safety and Quality in Medical Transport Systems: Creating an Effective Culture. Boca Raton, FL: CRC Press, 2019.

5. Kancherla BS, Upender R, Collen JF, et al. Sleep, fatigue and burnout among physicians: an American Academy of Sleep Medicine position statement. J Clin Sleep Med 2020;16:803-5.

6. Eden AR, Jabbarpour Y, Morgan ZJ, et al. Burnout Among Family Physicians by Gender and Age. J Am Board Fam Med 2020;33:355-6.

7. Goldberg DG, Soylu TG, Grady VM, et al. Indicators of Workplace Burnout Among Physicians, Advanced Practice Clinicians, and Staff in Small to Medium-Sized Primary Care Practices. J Am Board Fam Med 2020;33:378-85.

8. Dai $M$, Willard-Grace R, Knox M, et al. Team Configurations, Efficiency, and Family Physician Burnout. 
J Am Board Fam Med 2020;33:368-77.

9. Fiscella K, Mauksch L, Bodenheimer T, et al. Improving Care Teams' Functioning: Recommendations from Team Science. Jt Comm J Qual Patient Saf 2017;43:361-8.

10. Hettle D, Sutherland K, Miles E, et al. Cross-skill training to support medical redeployment in the COVID-19 pandemic. Future Healthc J 2020;7:e41-e44.

11. Thomas CH, Lankau MJ. Preventing burnout: The effects of LMX and mentoring on socialization, role stress, and burnout. Hum Resour Manage 2009;48:417-32.

12. Wanberg CR, Kammeyer-Mueller J, Marchese M. Mentor and protégé predictors and outcomes of mentoring in a formal mentoring program. J Vocat Behav 2006;69:410-23.

13. Merrill MD. First principles of instruction. Educ Technol Res Dev 2002;50:43-59.

doi: 10.21037/jhmhp-20-130

Cite this article as: Lai AY, Thomas SC, Sullivan EE, Fleuren BPI, Raj M, DePuccio MJ, Stephenson AL, McAlearney AS. COVID-19 and primary care physicians: adapting to rapid change in clinical roles and settings. J Hosp Manag Health Policy 2020;4:31. 